\title{
Key improvements in the simulation modelling for decision support systems developed in the EURANOS project
}

\author{
W. RASKOB ${ }^{1}$, F. GERING ${ }^{2}$
}

\begin{abstract}
One key issue in the EURANOS project was the methodological and technological improvement of decision support systems, in particular the RODOS system, with respect to the practical application in operational emergency centres. The development process was mainly driven by the result of demonstration activities and responses obtained from the end-users in the emergency centres. The intention of this article is to give an overview of all major work items which have been performed within EURANOS with the aim of improving decision support systems. More details about several of these activities can be found in separate articles in this issue.
\end{abstract}

\section{Introduction}

A significant work portion of the EURANOS project concentrated on the practical application of "state of the art" decision support systems and tools, in particular the RODOS system, in operational emergency centres. One key issue here was the methodological and technological improvement of RODOS on the basis of the results achieved under other EURANOS work packages - mainly the demonstration activities which aimed on testing the applicability of systems and tools under operational conditions - and the responses obtained from the end-users. One aim of this work was to ensure the wider acceptance and dissemination of decision support systems in Europe.

The further improvement of decision support systems was subdivided into three major topics:

1. estimation and management of the radiological situation and countermeasures in agricultural areas;

2. estimation and management of the radiological situation and countermeasures in inhabited areas;

3. enhancement of decision support systems.

The first two topics are covered in separate articles in this special issue. The work under these two topics concentrated on model enhancement and development, the

1 Karlsruhe Institute of Technology, Institut für Kern- und Energietechnik, Eggenstein-Leopoldshafen, Germany.

2 Bundesamt für Strahlenschutz, München-Neuherberg, Germany. 


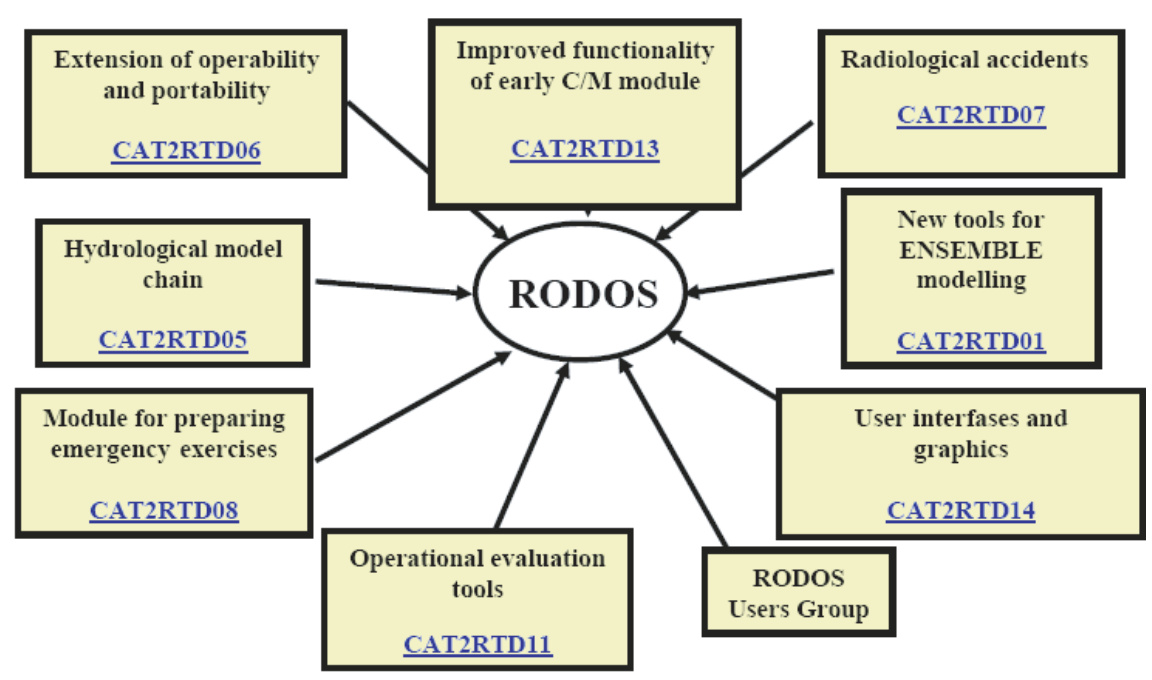

Figure 1 - EURANOS activities to improve the practical applicability of the RODOS system.

outcome (models and database) of these activities were integrated in the two decision support systems ARGOS and RODOS. In the following, an overview is presented for the third topic, the enhancement of decision support systems in general, which mainly concentrated on the RODOS system. This activity comprised of a number of individual work packages, which helped to make the RODOS system more user friendly, praxis oriented and functional with respect to a wider range of applications, see Figure 1. The intention of this article is to give an overview of all major work items which have been performed with the aim of improving the decision support system RODOS. More details about several of these activities can be found in separate articles in this issue.

All activities under the topic "enhancement of decision support systems" were strongly influenced by the demonstration projects organised within the EURANOS project and the feedback by the RODOS users group (RUG). The annual meeting of the RODOS users group turned out to be very effective in collecting user experiences from the past year and converting these experiences into direct feedback to the model and system developers of RODOS (which were always invited as hosts to these RUG meetings). The commitment of the RUG to the user-driven development of RODOS and the high interest in the project was demonstrated by immediately reactions of the RUG on newly developed EURANOS products and by the willingness of RUG members to spend resources not fully budgeted inside EURANOS. 


\section{Overview over activities for the enhancement of decision support systems}

\subsection{Development of ENSEMBLE-RODOS interface}

The ENSEMBLE system was developed between 2000 and 2003 supported by the European Commission. The system is a web-based platform for the collection, analysis and exchange of atmospheric dispersion predictions produced by a number of operational centres world-wide. Some users of the ENSEMBLE system reported during a demonstration project in EURANOS that dose calculations could be an important extension to the ENSEMBLE tool. Based on this an interface between ENSEMBLE and RODOS was developed, which allows to import ENSEMBLE results into RODOS and to calculate doses in RODOS based on the imported data. This interface is still somehow limited (e.g. performance, limited set of nuclides and covered models), but is sufficient for demonstration purposes. At the end of the project a decision was made not to continue work in this area and therefore the interface is neither a part of the RODOS nor of the ENSEMBLE system.

\subsection{Extension of the portability and operability of the RODOS system}

Several activities were bundled under the common title "Extension of the portability and operability of the RODOS system": mainly the migration of RODOS from HP-UX to Linux and various improvements of the atmospheric dispersion models for near and far range.

The aim of the migration of RODOS from HP-UX to Linux was to enable the use of RODOS in a wider range of hardware environments, especially since emergency centres are often relatively strict in their regulations regarding the hardware environment in the centre and thus systems to be run operationally in the centres often have to be capable to run in a given hardware environment (and not that the regulations are adapted to the need of certain systems). The migration process was started with an external review of the migration concept. Later the migration was carried through stepwise, starting with the system core and followed by the models. In addition to the standard Linux version, a so called "virtual machine" could also be delivered. This free virtualisation software allows the distribution of the system in a pre-configured stage with complete network functionalities and running under the most common host operation systems in particular also under Windows XP. Within a short time after the Linux version of RODOS became available, most users of the system switched over to Linux and this increased in many cases the performance of the system significantly (mainly because hardware costs for Linux systems are much lower than hardware costs for HP-UX systems and number crunching performance is higher). 
The long-range atmospheric dispersion model in RODOS (MATCH) was enhanced to deal with all source terms stored in the RODOS system and to consider additional information provided nowadays from numerical weather prediction output. The MATCH model was also enhanced to read data from various numerical weather services providing data for the long range forecast. This comprises the ECMWF forecast system located in Reading and a slightly modified Aladin output.

The mass-consistent wind field model - which delivers data to all dispersion models in RODOS - was finalised and implemented into RODOS as a separate module that is executed as part of the meteorological pre-processing. The software code of the mass-consistent wind field model has been based on the existing diagnostic meteorological model ADREA (e.g. Venetsanos, 2004), with adaptations for the terrain-following grid of the meteorological pre-processor. The function of the mass-consistent wind field model is to correct the wind velocity components, which are produced through interpolation by the meteorological preprocessor (the "first-guess" field), taking into account the topography (i.e., the ground slopes), in order to assure that the air mass is conserved in every computational cell. The mass-consistent wind field model is a particularly useful feature in case of highly complex terrain that modifies significantly the wind field. The implemented model is 3-dimensional, i.e., it calculates a vertical wind velocity component, in addition to the modification of the horizontal components.

The particle model DIPCOT for complex terrain was integrated into RODOS in its interactive and automatic versions. The Lagrangian model includes a module for the random motion of the pollutant particles. For the concentration calculations both a Gaussian kernel and a "box-counting" scheme are included.

Comparison calculations between the three atmospheric dispersion models in RODOS (ATSTEP, RIMPUFF and DIPCOT) were performed, demonstrating that all three provide similar results for simple meteorological conditions. For complex situations, DIPCOT differs and represents the meteorology in a more consistent way, in particular when there is wind sheer with height. During the comparison calculations differences between RIMPUFF and ATSTEP were observed. The consideration of the change in the wind direction with height was the reason for this difference. To harmonise both models, the approach in ATSTEP was adapted to the one in RIMPUFF and now both models behave quite similar.

Work was also performed to enhance the uncertainty estimation of the numerical weather data provided by national weather services allowing to better quantify uncertainties between local measurements and calculations. Diagnostic and prognostic weather data for three months and for 7 sites in Germany and 
Denmark have been compared and uncertainty factors can be now estimated for the usage in the data assimilation software of the atmospheric dispersion models.

\subsection{Enhancement of RODOS for radiological emergencies}

The decision support system RODOS has been developed as a decision-aiding tool for off-site emergency management caused by airborne accidental releases from nuclear installations, in particular nuclear power plants ("nuclear accidents"). During the past years it became more and more obvious, that end-users are interested in extending the range of applicability of RODOS to all kinds of events, accidents, or attacks, involving radioactive material ("radiological emergencies", e.g. attacks with radiological dispersal devices - "dirty bombs" -, accidents during the transport of radioactive material, re-entry of a satellite or spacecraft with radioactive material on board). For this reason one activity within EURANOS was to enhance RODOS for radiological emergencies. To realise this, different radiological emergency scenarios were surveyed, the principle applicability of RODOS for these scenarios was investigated and necessary model modifications or database extensions were described. In a second step a set of additional radionuclides was implemented in the data base and an explosion sub-module was developed, which delivers input data to atmospheric dispersion modules. The explosion sub-module describes the initial blast of an explosion based on the approach adopted in HOTSPOT (LLNL, 2005). Additionally the graphical user interface was extended to cover all necessary input for radiological emergencies.

\subsection{Development of a flexible module for preparing emergency exercises}

In emergency centres operating decision support systems the daily use of the system typically concentrates on training, functional demonstration, tests and (sometimes) exercises and exercise preparation. Preparation of e.g. full-scale exercises or even exercises involving only automated monitoring networks can add-up to a large amount of work for the personnel in emergency centres. To facilitate this work, a tool for preparing emergency exercises was developed for RODOS within the EURANOS projects. Due to upcoming new priorities during the project - basically the re-engineering of the full RODOS system - less work than originally planned was performed in this activity, but nevertheless a tool with basic functionality was developed: monitoring data can be simulated based on an atmospheric dispersion calculation, including data for fixed monitoring networks and mobile units. The simulated monitoring data includes gamma dose rate, nuclide-specific ground contamination and concentration in air near ground (time sequences for all data). The user input is being carried out with a graphical user interface which is fully compatible to the new graphical user interface for RODOS, 
RoLite. Only a small set of input data is required to characterize the chosen radiological scenario (e.g. meteorological data, source term, locations for monitoring data).

\subsection{Improvement of the stability of the RODOS system}

From an operational point of view one of the most important improvements of the RODOS system during the EURANOS project was the enhancement of the stability of long-term calculations with real-time monitoring data. In one mode of operation - called "automatic mode" - RODOS calculates (typically) every 10 minutes a new time interval for the atmospheric dispersion using on-line realtime monitoring data (meteorological and release data) from a nuclear power plant. In case of an upcoming release or during a release the user can operate RODOS in this mode continuously, thus monitoring the current situation at the NPP with the most current data being available. Under emergency conditions such a situation can last up to several days, thus demanding a very reliable and robust system. Users of the RODOS system noted before the start of EURANOS, that calculations with real-time monitoring data sometimes stopped due to incomplete monitoring data. Such situations with incomplete data sets can easily occur since the priority for providing real-time data is put on rapidness of data provision, not on compensation, error-correction or validation mechanisms. Furthermore, some of the measurement methods - like the SODAR measurements - can not provide reliable data under certain (meteorological) conditions. As a result of this it was decided that within the EURANOS project the stability of operation with real-time data should be significantly increased. The improvements were introduced in an iterative process, where mainly the emergency centre of Lower Saxony, Germany, conducted intense long-term tests with artificially introducing all variants of data losses and reporting all problems directly to the system developers, who immediately analysed the problem and delivered fixes for the system back to the test team. After a six month period with more than 200 test cases the stability of RODOS when operating with real-time data was significantly increased, being demonstrated by several final test runs with many days of continuous, error-free operation.

\subsection{Further enhancement of evaluation tools in decision support systems for operational applicability}

Work under this activity aimed on improving the decision aiding component of RODOS and focused on the development of a concept for the visualisation of uncertainties and how sequential decision making may influence the selection of the optimal countermeasure strategy. A decision making process supported by 
RODOS is subject to various sources of uncertainty: According to their respective source, a distinction can be made between "data uncertainties" (uncertainties of the input data to a model), "parameter uncertainties" (uncertainties related to the model parameters) and "model uncertainties" (uncertainties resulting from the fact that models are ultimately only simplifications/approximations of reality). However, the different types of uncertainty are of varying importance in the different phases of emergency management. In the early phase, the RODOS calculations are strongly affected by the uncertain input data, especially the source term and the wind fields. Within EURANOS a concept was proposed for handling the uncertainties associated with the input data of the RODOS calculations and the visualisation of their impact on the results of the atmospheric dispersion calculations.

\subsection{Guidance on monitoring and data assimilation}

In a nuclear or radiological emergency the bases for decisions are often predictions produced with decision support systems (DSS). Actual radiation measurement data should be used to improve the reliability of the predictions. Data assimilation is an important link between model calculations and measurements. Different aspects have to be taken into account for the optimal use of the data assimilation technique. These aspects were investigated in the EURANOS work package "Guidance on monitoring and data assimilation" and the findings were documented in a EURANOS report (European Commission, 2006). The situation during and after an accident was analysed and a list of recommendations was given that can help modellers to take into account the measurements that are best suited for data assimilation. More details can be found in a separate article in this issue.

\subsection{User friendly application of RODOS}

During the development of RODOS over more than a decade the system was constantly enhanced by adding additional features or models. The increasing complexity of the system led to an increasing complexity of the user interface. Often the original design of the user interface proved to be inadequate or not well suited for the handling of new features or models and compromises or a "workaround" had to be introduced. During this process the - always existing - interests of the RODOS users in a modern and appropriate user interface - suited for the use even under emergency conditions - were not appropriately considered. For this reason a new, user friendly and light-weight access to the RODOS functionalities was strongly requested by the end users from the beginning of the EURANOS project. The RODOS users group RUG developed detailed requirements for the user interface (even including proposals for the layout and content of the interface) 
and communicated this within several meetings with the system developers. The major requirements were:

- The user interface should be self-explaining, enabling the use without constant training and even under emergency conditions.

- Rarely used features should be initialised with default values and hidden in normal application.

- The user interface should be realised as a separated application establishing an open interface to the RODOS core. The user interface should be easy to transfer between different operating systems and also between the desktop and the web application.

- The user interface should not follow the structure of the underlying models anymore, but should be flexible to consider the user choice for results of interest (i.e. only such user input required to calculate the requested results should be demanded by the system).

- The user interface should be based upon well established and state-of-the-art IT-standards to assure long-term support and acceptance.

These requirements were fulfilled by the design and implementation of a new user interface named RODOS-Lite. The main purpose of this new user interface is to gently lead operators through a sequential line of input masks, hiding rarely used parameters (defaults are set) from the operator but still providing the possibility to access them if necessary. Additionally, the interface is strongly safeguarded to prevent operators from entering wrong input values. This is achieved by systematically checking input values and even suggesting occasionally best estimate parameters.

RODOS-Lite was implemented using the Java programming language. Java is available for almost every major computer architecture and operating system including HP-UX, Linux and the Windows family. An open interface between the standalone RODOS-Lite application and the RODOS core is realised through file exchange of XML files. This open interface enables third party tools to access the RODOS core components, allows to run the system without user interface (e.g. with pre-defined XML files) or to loosely couple the RODOS system with other emergency management tools (e.g. electronic reporting systems).

The RODOS-Lite user interface has a Windows-like look and feel and is organized in a main section and a smaller information section, where basic parameters are permanently displayed. The main section is build of separate tabs <reiter $>$, each including a logical block of input fields: for example, one $<$ reiter $>$ including all input fields related to the source term definition, another one for the definition of meteorological parameters, etc. Each < reiter $>$ has to be processed and confirmed to allow advancing to the next one. 


\subsection{Re-engineering of RODOS}

One of the major objectives of the EURANOS project was to improve the RODOS system in its operational applicability. The RODOS Users Group RUG concluded during the first phase of the EURANOS project that a significant improvement could be only achieved through a complete software re-engineering of the existing system. The project management team accepted this recommendation and initiated and organised the development process. The decision on the re-engineering caused substantial modifications in the work program. The RUG laid down their requirements for the re-engineering of the RODOS system in a long "wish list", in which priorities for the various requirements were provided. General demands were listed and more detailed requests were developed for a list of 16 use cases, which described typical applications of the system in an emergency centre. Especially important for the RUG were the following topics to be considered in the re-engineering:

- Use RODOS-Lite as user interface where applicable (see section above).

- Graphical representation of results that meets user requirements (e.g. integration of state-of-the-art GIS subsystem supporting common GIS standards, multi-lingual, annotated, etc.).

- Easy system administration and low maintenance costs.

- Preserve main computational models of RODOS.

- The system should be cross-platform with running on Windows, Linux, possibly MacOS.

- The system should have a distributed architecture to be applicable within different environments.

- Technical support including hot-line, web page (properly managed, supervised and kept actual, with notification on news, etc.) for download both updates, patches, new versions etc. and guides and manuals, FAQ section.

- Easy way of integrating external simulation modules in a framework with clearly defined interfaces.

The RUG also requested that intermediate prototypes should be released for immediate testing. With this prototyping the RUG assured that new features introduced in one prototype could be immediately tested and feedback from the tests could be used in the development of the next prototype. This feedback loop via the numerous prototypes released within the two years of the re-engineering guaranteed that the development followed the requests and recommendations of the operational end users.

Being a Java based product, the new RODOS version named JRODOS introduces a cross-platform solution capable to run on most operation systems, including Windows and the main UNIX derivates. The re-engineered system 
preserves all computational models from RODOS, adding a powerful GIS support and applying modern database technologies with flexible configuration possibilities. JRODOS import functionality includes the support of EURDEP measurement files and GRIB meteo data. The system operates successfully on Windows (NT based versions from Windows 2000 to Windows 7) and UNIX machines (OpenSuSe, Ubuntu, Solaris), both x86 and x64. Computational models are integrated into JRODOS as plug-ins. This allows easy system expansion and facilitates independent development of new models outside the system. Any RODOS further development will be realized in the JRODOS version aiming to have this as the main operational version for the next 10 years.

The JRODOS user interface has a modern look and feel and is a convenient tool for the communication with the operator. It is implemented as static window frame with several components. The central frame is a tabbed pane which serves for data representation/visualization of various types (scalar values, maps, time plots, tables, text, histograms) and collection of user input for the simulation models, which is realised by the integration of the newly developed and highly appreciated RODOS-Lite user interface (see section above). Additionally a web interface has been implemented which allows limited access to JRODOS functionalities and the project database via a web browser. It is possible to request calculation services within the web browser by using the RODOS-Light user interface.

JRODOS uses the open source PostgreSQL database engine for storing data. The engine can be implemented and used on a machine physically separated from the core JRODOS one, allowing several Clients to be connected to it. Thus, all the system data can be shared between different JRODOS Clients. To process and display geographical data, JRODOS uses the Java open source Geotools library, a powerful GIS system, which supports spatial data representation and analysis as well as common data standards (.shp, .tiff) and technologies (WMS). In addition it is possible to use Google Maps as a map background in JRODOS. Results of the model calculations can be combined in so called Reports to be forwarded to the decision making team. For the generation of these reports, JRODOS uses the opensource Pentaho reporting engine. Generated reports can be printed, saved and stored in pdf, xls, html, rtf, text formats. The system allows manual and automatic report generation.

\section{Conclusions}

Within the five years of the EURANOS project, new simulation models have been developed and other existing models further enhanced. Both has been based either on findings from the demonstration projects or based on user feedback. Improvements were realised in the near and far range atmospheric dispersion 
capabilities, in the field of monitoring and data assimilation, by a first extension to radiological emergencies, related to exercises and in the decision aiding component. Furthermore, the operational applicability of the RODOS system was strongly enhanced by introducing a new user interface and finally by the reengineering of the system. This will allow to operate the system on a modern IT-basis for the next decade.

\section{REFERENCES}

European Commission (2006) "Guidance on model adaptation driven by monitoring data", the work package CAT1RTD12 in the integrated project European Approach to Nuclear and Radiological Emergency Management and Rehabilitation Strategies (EURANOS) of the Sixth Framework Programme of the European Union (Contract number: FI6R-CT-2004-508843).

LLNL (Lawrence Livermore National Laboratory) (2005) Hotspot Health Physics Code for the PC. Available at http://www.llnl.gov/nai/technologies/hotspot/

Venetsanos A.G., Bartzis J.G., Andronopoulos S. (2004) One Equation Turbulence Modeling for Atmospheric and Engineering Applications, Boundary-Layer Meteorol. 113, 321-346. 